\title{
A Dimensionality Result for Multiple Homography Matrices
}

\author{
Wojciech Chojnacki Anton van den Hengel \\ School of Computer Science, The University of Adelaide \\ Adelaide, SA 5005, Australia \\ \{wojciech. chojnackilanton. vandenhengel\}@adelaide.edu.au
}

\begin{abstract}
It is shown that the set of all I-element collections of interdependent homography matrices describing homographies induced by I planes in the $3 D$ scene between two views has dimension $4 I+7$. This improves on an earlier result which gave an upper bound for the dimension in question, and solves a long-standing open problem. The significance of the present result lies in that it is critical to the identification of the full set of constraints to which collections of interdependent homography matrices are subject, which in turn is critical to the design of constrained optimisation techniques for estimating such collections from image data.
\end{abstract}

\section{Introduction}

The estimation of a single homography matrix from image measurements is an important step in $3 \mathrm{D}$ reconstruction, mosaicing, camera calibration, metric rectification and other tasks [6]. For some applications, like non-rigid motion detection $[9,7]$ for instance, a whole array of homography matrices, all intrinsically interconnected, are required. The matrices must satisfy the consistency constraints implied by the rigidness of the motion and the scene. One of the fundamental problems in estimating multiple homography matrices is to find a way to enforce these underlying consistency constraints-a task reminiscent of that of enforcing the rank-two constraint in the case of the fundamental matrix estimation [6].

As a rule, the consistency constraints are available only in implicit form. The conventional approach for dealing with such constraints is to evolve a derivative family of explicit constraints. These new constraints are typically more relaxed than the original ones. Adhering to this methodology, Shashua and Avidan [8] found that homography matrices induced by four or more planes in a 3D scene appearing in two views span a 4-dimensional linear subspace. Chen and Suter [2] derived a set of strengthened constraints for the case of three or more homographies in two views.
Zelnik-Manor and Irani [9] have shown that another rankfour constraint applies to a set of so-called relative homographies generated by two planes in four or more views. These latter authors also derived constraints for larger sets of homographies and views.

Once isolated, the explicit constraints can be put to use in a procedure whereby first individual homography matrices are estimated from image data, and then the resulting estimates are upgraded to matrices satisfying the constraints. Following this pattern, Shashua and Avidan as well as Zelnik-Manor and Irani used low-rank approximation under the Frobenius norm to enforce the rank-four constraint. Chen and Suter enforced their set of constraints also via low-rank approximation, but then employed the Mahalanobis norm with covariances of the input homographies. All of these estimation procedures produce matrices that satisfy only the derivative constraints so their true consistency cannot be guaranteed.

The constraints can also be taken care of directly by recoursing to a parametrisation of the set of all intervening homography matrices. Following this path, Chojnacki et al. [3] employed a specific parametrisation and a specific cost function to develop an upgrade procedure based on unconstrained optimisation. While all implicit constraints were enforced in this way, there remained the problem of finding a good initialisation for the underlying iterative optimisation scheme. One alternative to the initialisation procedure proposed in [3] involves the application of a computationally efficient and statistically accurate constrained optimisation technique. To devise such a method, however, one first needs to identify all the explicit constraints.

The present paper, theoretical in nature, is a step towards the identification of these explicit constraints. Its main contribution is in revealing the dimension of the set of all interdependent homography matrices in the case when the homographies described by these matrices are induced by multiple planes in a rigid 3D scene in two views. As an immediate consequence, the number of explicit constraints is also derived. It is a matter of further work to exploit this latter information to delineate the constraints explicitly. 


\section{Prerequisites}

We start with some prerequisites necessary for establishing our main result. These will include a presentation of a specific way in which sets of homography matrices will be organised, and basic background material concerning the concept of dimension.

\subsection{Multi-homography matrices}

Hereafter we shall consider exclusively the case of homographies induced by multiple planes in the 3D scene between two views. In our analysis, it will prove convenient to convert sets of interdependent homography matrices into specific matrices. These matrices will be different from the horizontal or vertical concatenations of the individual homography matrices forming each set.

Denote by $\mathbb{R}$ the set of real numbers and by $\mathbb{R}^{m \times n}$ the set of real $m \times n$ matrices. Suppose that $\mathbf{P}_{1}=\left[\mathbf{I}_{3}, \mathbf{0}\right]$ and $\mathbf{P}_{2}=$ $[\mathbf{A},-\mathbf{b}]$ are two fixed camera matrices. Here $\mathbf{I}_{3}$ is the $3 \times 3$ identity matrix, $\mathbf{0}$ is the length- 3 zero vector, $\mathbf{A} \in \mathbb{R}^{3 \times 3}$, and $\mathbf{b} \in \mathbb{R}^{3}$. Suppose, moreover, that a set of $I$ planes in a 3D scene have been selected. Given $i=1, \ldots, I$, let the $i$-th plane from this set be characterised by a length- 4 vector $\left[\mathbf{v}_{i}^{\top}, w_{i}\right]^{\top}$ with $\mathbf{v}_{i} \in \mathbb{R}^{3}$ and $w_{i} \in \mathbb{R}$. For each $i=1, \ldots, I$, the $i$-th plane gives rise to a planar homography $\mathbf{H}_{i}$ from view $\mathbf{P}_{2}$ to view $\mathbf{P}_{1}$ described by the $3 \times 3$ matrix

$$
\mathbf{H}_{i}=w_{i} \mathbf{A}+\mathbf{b v}_{i}^{\top} .
$$

For each $i=1, \ldots, I$, let $\mathbf{h}_{i}=\operatorname{vec}\left(\mathbf{H}_{i}\right)$, where vec denotes column-wise vectorisation, and let $\mathbf{H}$ be the $9 \times I$ matrix given by

$$
\mathbf{H}=\left[\mathbf{h}_{1}, \ldots, \mathbf{h}_{I}\right] .
$$

Henceforth any $\mathbf{H}=\mathbf{H}\left(\mathbf{A}, \mathbf{b}, \mathbf{v}_{1}, \ldots, \mathbf{v}_{I}, w_{1}, \ldots, w_{I}\right)$ of this form will be referred to as a multi-homography matrix. The set of all multi-homography matrices will be denoted by $\mathcal{H}$.

\subsection{Dimension}

There are various concepts of dimension used in mathematics, not all of them equivalent. Intuitively, the dimension of a set is the least number of parameters needed to locally describe this set. Here we shall use the notion of dimension for semi-algebraic sets. A semi-algebraic set is a subset of the real $n$-dimensional space $\mathbb{R}^{n}$ which can be written as a finite union of subsets defined by a finite conjunction of polynomial equalities and inequalities. Our interest in semialgebraic sets stems from the link between such sets and the polynomial images of $\mathbb{R}^{n}$.

Given positive integers $m$ and $n$, a map $\mathbf{f}=$ $\left[f_{1}, \ldots, f_{n}\right]^{\top}: \mathbb{R}^{m} \rightarrow \mathbb{R}^{n}$ is said to be polynomial if the functions $f_{i}$ are polynomials in $x_{1}, \ldots, x_{m}$. The celebrated Tarski-Seidenberg theorem [1] ensures that the image of

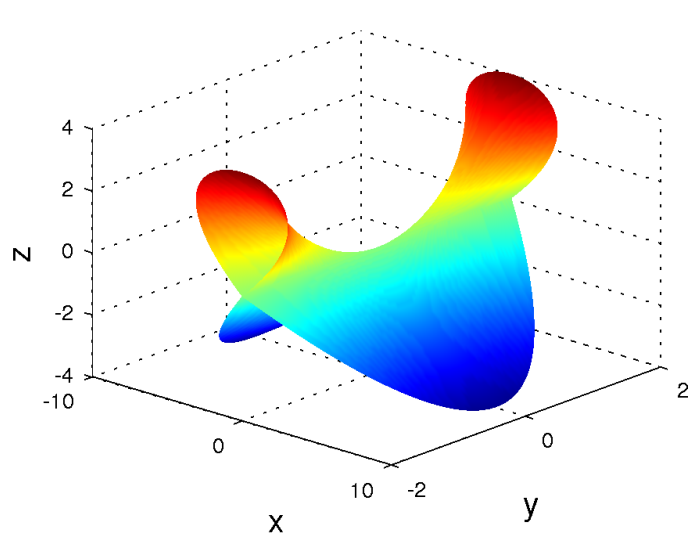

Figure 1. Plot of a portion of the variety $x^{2}-y^{2} z^{2}+z^{3}=0$.

any polynomial map $\mathbf{f}: \mathbb{R}^{m} \rightarrow \mathbb{R}^{n}$ is a semi-algebraic subset of $\mathbb{R}^{n}$.

Some semi-algebraic sets are smooth manifolds and some are not. Consider, for example, the image in $\mathbb{R}^{3}$ of $\mathbb{R}^{2}$ by the polynomial map

$$
[t, u]^{\top} \mapsto\left[t\left(u^{2}-t^{2}\right), u, u^{2}-t^{2}\right]^{\top} .
$$

It coincides with the variety $x^{2}-y^{2} z^{2}+z^{3}=0$. This variety is not a smooth manifold because, locally, at each point of the $y$-axis other than the origin, the surface looks like the intersection of two smooth manifolds-see Figure 1.

Any semi-algebraic set is locally, on a dense open subset, a submanifold embedded in the ambient space. One can define the dimension of a semi-algebraic set to be the largest dimension at points at which it is a submanifold.

\section{Main result}

As it turns out, our set of interest $\mathcal{H}$ is a polynomial image of $\mathbb{R}^{4 I+12}$ (see Section 4.1 for a full explanation). Consequently, $\mathcal{H}$ is semi-algebraic and one can speak about its dimension. Our main result is that the dimension of $\mathcal{H}$ is equal to $4 I+7$. We shall split the argument justifying this result into two parts, corresponding to two inequalities: $\operatorname{dim} \mathcal{H} \leq 4 I+7$ and $\operatorname{dim} \mathcal{H} \geq 4 I+7$. The first inequality has already surfaced in the literature [4], but the derivation of it that we present here is in some aspects new. The second inequality is novel and constitutes the main contribution of the paper.

\section{Upper dimension bound}

We first show that $\operatorname{dim} \mathcal{H} \leq 4 I+7$. With a view to gaining some historical perspective, we start by presenting some weaker bounds obtained earlier and only then do we derive the ultimate bound. 


\subsection{Initial upper bounds}

Any multi-homography matrix $\mathbf{H}$ is naturally expressed in terms of an array of parameters

$$
\boldsymbol{\omega}=\left(\mathbf{A}, \mathbf{b}, \mathbf{v}_{1}, \ldots, \mathbf{v}_{I}, w_{1}, \ldots, w_{I}\right),
$$

where $\mathbf{A} \in \mathbb{R}^{3 \times 3}, \mathbf{b} \in \mathbb{R}^{3}, \mathbf{v}_{i} \in \mathbb{R}^{3}$, and $w_{i} \in \mathbb{R}$. More specifically, if $\boldsymbol{\Pi}(\boldsymbol{\omega})$ is the $3 \times 3 I$ matrix given by

$$
\boldsymbol{\Pi}(\boldsymbol{\omega})=\left[\boldsymbol{\Pi}_{1}(\boldsymbol{\omega}), \ldots, \boldsymbol{\Pi}_{I}(\boldsymbol{\omega})\right],
$$

where

$$
\boldsymbol{\Pi}_{i}(\boldsymbol{\omega})=w_{i} \mathbf{A}+\mathbf{b v}_{i}^{\top}
$$

for each $i=1, \ldots, I$, then

$$
\mathbf{H}=r(\boldsymbol{\Pi}(\boldsymbol{\omega})) .
$$

Here $r$ denotes the mapping

$$
\left[\mathbf{M}_{1}, \ldots, \mathbf{M}_{I}\right] \mapsto\left[\operatorname{vec}\left(\mathbf{M}_{1}\right), \ldots, \operatorname{vec}\left(\mathbf{M}_{I}\right)\right]
$$

When the $\mathbf{M}_{i}$ are $3 \times 3$ matrices, $r(\cdot)$ can be identified with MATLAB's reshape $(\cdot, 9, I)$ operator. While the array $\boldsymbol{\omega}$ has entries of different types, it can always be reshaped to a length- $(4 I+12)$ vector, for example

$$
\left[\operatorname{vec}(\mathbf{A})^{\top}, \mathbf{b}^{\top}, \mathbf{v}_{1}^{\top}, \ldots, \mathbf{v}_{I}^{\top}, w_{1}, \ldots, w_{I}\right]^{\top},
$$

and be viewed as an element of $\mathbb{R}^{4 I+12}$. Consequently, the set $\Omega$ of all arrays $\boldsymbol{\omega}$ as above has dimension $4 I+12$. As the dimension of the set of multi-homography matrices is no bigger than the dimension of its associated set of parameter arrays, it immediately follows that $\operatorname{dim} \mathcal{H} \leq 4 I+12$.

This estimate can be further refined to the inequality $\operatorname{dim} \mathcal{H} \leq 4 I+10$ [2]. Indeed, since, with $\mathbf{a}=\operatorname{vec}(\mathbf{A})$,

$$
\mathbf{h}_{i}=w_{i} \operatorname{vec}(\mathbf{A})+\operatorname{vec}\left(\mathbf{b} \mathbf{v}_{i}^{\top}\right)=w_{i} \mathbf{a}+\left(\mathbf{I}_{3} \otimes \mathbf{b}\right) \mathbf{v}_{i}
$$

it follows that

$$
\mathbf{H}=\mathbf{H}^{\prime}+\mathbf{H}^{\prime \prime},
$$

where

$$
\mathbf{H}^{\prime}=\left[w_{1} \mathbf{a}, \ldots, w_{I} \mathbf{a}\right]=\mathbf{a w}^{T}, \quad \mathbf{w}=\left[w_{1}, \ldots, w_{I}\right]^{\top}
$$

and

$$
\begin{gathered}
\mathbf{H}^{\prime \prime}=\left[\left(\mathbf{I}_{3} \otimes \mathbf{b}\right) \mathbf{v}_{1}, \ldots\left(\mathbf{I}_{3} \otimes \mathbf{b}\right) \mathbf{v}_{I}\right]=\left(\mathbf{I}_{3} \otimes \mathbf{b}\right) \mathbf{V}, \\
\mathbf{V}=\left[\mathbf{v}_{1}, \ldots, \mathbf{v}_{I}\right] .
\end{gathered}
$$

Clearly, $\mathbf{H}^{\prime}$ is a rank-one $9 \times I$ matrix. Corresponding to $\mathbf{H}^{\prime \prime}$, define a $3 \times 3 I$ matrix $\mathbf{H}_{0}^{\prime \prime}$ by

$$
\mathbf{H}_{0}^{\prime \prime}=\left[\mathbf{b} \mathbf{v}_{1}^{\top}, \ldots, \mathbf{b} \mathbf{v}_{I}^{\top}\right]=\mathbf{b}\left[\mathbf{v}_{1}^{\top}, \ldots, \mathbf{v}_{I}^{\top}\right] .
$$

The factorisation in the rightmost term shows that $\mathbf{H}_{0}^{\prime \prime}$ has rank one. Now, $\mathbf{H}^{\prime \prime}=r\left(\mathbf{H}_{0}^{\prime \prime}\right)$, and so

$$
\mathbf{H}=\mathbf{H}^{\prime}+r\left(\mathbf{H}_{0}^{\prime \prime}\right) \text {. }
$$

Denote by $\mathbb{R}_{k}^{m \times n}$ the set of real $m \times n$ matrices of rank at most $k$. It is well known that $\mathbb{R}_{k}^{m \times n}$ is a $k(m+n-$ $k$ )-dimensional variety in $\mathbb{R}^{m \times n}$ [5]. Given that $\mathbb{R}_{1}^{9 \times I}$ and $\mathbb{R}_{1}^{3 \times 3 I}$ to which $\mathbf{H}^{\prime}$ and $\mathbf{H}_{0}^{\prime \prime}$ belong, respectively, have their corresponding dimensions equal to $I+8$ and $3 I+2, \mathbf{H}$ can be expressed in terms of $(I+8)+(3 I+2)=4 I+10$ parameters. Thus $\operatorname{dim} \mathcal{H} \leq 4 I+10$.

\subsection{Ultimate upper bound}

A still better, in fact optimal, upper estimate of the dimension of $\mathcal{H}$ is $\operatorname{dim} \mathcal{H} \leq 4 I+7$ [4]. We shall derive it by exploiting the fact there are many different parameter arrays describing the same multi-homography matrix. Our derivation will pursue a slightly different path than that taken in [4].

For each matrix

$$
\mathbf{C}=\left[\begin{array}{llll}
\alpha & 0 & 0 & c_{1} \\
0 & \alpha & 0 & c_{2} \\
0 & 0 & \alpha & c_{3} \\
0 & 0 & 0 & \beta
\end{array}\right]
$$

where $\alpha, \beta \in \mathbb{R} \backslash\{0\}$ and $\mathbf{c}=\left[c_{1}, c_{2}, c_{3}\right]^{\top} \in \mathbb{R}^{3}$, let $\tau_{\mathbf{C}}$ be the transformation of $\Omega$ into itself given by

$$
\begin{aligned}
\tau_{\mathbf{C}}(\boldsymbol{\omega})= & \left(\beta \mathbf{A}+\mathbf{b c}^{\top}, \alpha \mathbf{b}\right. \\
& \alpha^{-1} \mathbf{v}_{1}-\alpha^{-1} \beta^{-1} \mathbf{c}, \ldots, \alpha^{-1} \mathbf{v}_{I}-\alpha^{-1} \beta^{-1} \mathbf{c} \\
& \left.\beta^{-1} w_{1}, \ldots, \beta^{-1} w_{I}\right)
\end{aligned}
$$

With the matrix composition as group operation and with the $4 \times 4$ identity matrix $\mathbf{I}_{4}$ as neutral element, the set $G$ of all matrices $\mathbf{C}$ as above is a group. Denote by $\operatorname{Aut}(\Omega)$ the set of all one-to-one transformations of $\Omega$. Under the composition of mappings as group operation and with the identity mapping of $\Omega$ as neutral element, $\operatorname{Aut}(\Omega)$ is a group. It is readily verified that the function $\tau: \mathbf{C} \mapsto \tau_{\mathbf{C}}$ maps $G$ into $\operatorname{Aut}(\Omega)$ (so that each $\tau_{\mathbf{C}}$ is a bijection) and is a homomorphism:

$$
\tau_{\mathbf{C}} \tau_{\mathbf{C}^{\prime}}=\tau_{\mathbf{C C}^{\prime}}, \quad \tau_{\mathbf{C}}^{-1}=\tau_{\mathbf{C}^{-1}}
$$

for any $\mathbf{C}, \mathbf{C}^{\prime} \in G$. The critical property of the $\tau_{\mathbf{C}}$ 's is that any of these transformations leaves all the homography matrices unchanged:

$$
\boldsymbol{\Pi}\left(\tau_{\mathbf{C}}(\boldsymbol{\omega})\right)=\boldsymbol{\Pi}(\boldsymbol{\omega})
$$

for each $\boldsymbol{\omega} \in \Omega$. Thus the $\tau_{\mathbf{C}}$ 's constitute a group of internal symmetries related to the freedom of choice of parameter arrays. The fact that $\tau$ is a homomorphism can be phrased as saying that $\tau$ is a representation of $G$ in the gauge group. The latter group comprises all transformations $\gamma$ in $\operatorname{Aut}(\Omega)$ such that $\boldsymbol{\Pi}(\gamma(\boldsymbol{\omega}))=\boldsymbol{\Pi}(\boldsymbol{\omega})$ for each $\boldsymbol{\omega} \in \Omega$. Under the equivalence relation in which $\boldsymbol{\omega}, \boldsymbol{\omega}^{\prime} \in \Omega$ 
are regarded as equivalent whenever $\boldsymbol{\omega}^{\prime}=\tau_{\mathbf{C}}(\boldsymbol{\omega})$ for some $\mathbf{C} \in G$, the set $\Omega$ is partitioned into classes of intrinsically equivalent parameter arrays, each class representing exactly one underlying multi-homography matrix. Consequently, $\operatorname{dim} \mathcal{H} \leq \operatorname{dim} \Omega-\operatorname{dim} G=(4 I+12)-5=4 I+7$.

\section{Lower dimension bound}

Here we show that $\operatorname{dim} \mathcal{H} \geq 4 I+7$. This together with the last result of the previous section will imply that $\operatorname{dim} \mathcal{H}=4 I+7$. The lower bound on the dimension of $\mathcal{H}$ is obtained through analysis of the linearisation of a specific parametrisation of $\mathcal{H}$. The use of a differential method makes the highly non-linear problem of determining redundancies in parametrisation amenable to a simpler, linear technique.

Let $\Omega_{0}$ be the set of those $\omega$ in $\Omega$ for which

$$
\|\mathbf{b}\|^{2}=\mathbf{b}^{\top} \mathbf{b}=1 .
$$

As pointed out earlier, $\Omega$ is essentially identical to the Euclidean space $\mathbb{R}^{4 I+12}$. Accordingly, $\Omega_{0}$ can be viewed as a hypersurface in $\mathbb{R}^{4 I+12}$. Consider the restriction $\bar{\Pi}$ of the map $\Pi$ to $\Omega_{0}$,

$$
\bar{\Pi}: \Omega_{0} \rightarrow \mathbb{R}^{3 \times 3 I}, \quad \bar{\Pi}(\boldsymbol{\omega})=\Pi(\boldsymbol{\omega}) \quad \text { for } \boldsymbol{\omega} \in \Omega_{0} .
$$

Note that the image $\bar{\Pi}\left(\Omega_{0}\right)$ of $\Omega_{0}$ by $\bar{\Pi}$ is identical to the image $\Pi(\Omega)$ of $\Omega$ by $\Pi$. Indeed, given $\omega \in \Omega$, the right-hand side of (1) does not change if $\boldsymbol{\omega}$ is replaced by $\boldsymbol{\omega}_{0} \in \Omega_{0}$ defined as the modification of $\boldsymbol{\omega}$ in which $\|\mathbf{b}\|^{-1} \mathbf{b}$ is substituted for $\mathbf{b}$ and, for each $i=1, \ldots, I,\|\mathbf{b}\| \mathbf{v}_{i}$ is substituted for $\mathbf{v}_{i}$, the rest of the entries of $\boldsymbol{\omega}$ remaining unaltered. Now, $\bar{\Pi}\left(\Omega_{0}\right)$ coincides with the image $r^{-1}(\mathcal{H})$ of $\mathcal{H}$ by the inverse mapping $r^{-1}$ to $r$-see (2). As $r$ is a oneto-one smooth mapping, it and its inverse do not change the dimension of sets that they transform. In particular,

$$
\operatorname{dim} \mathcal{H}=\operatorname{dim} r^{-1}(\mathcal{H})=\operatorname{dim} \bar{\Pi}\left(\Omega_{0}\right) .
$$

Thus to complete the argument, it suffices to show that $\operatorname{dim} \bar{\Pi}\left(\Omega_{0}\right) \geq 4 I+7$.

Let $\mathrm{d} \overline{\boldsymbol{\Pi}}_{\boldsymbol{\omega}}$ denote the differential of $\overline{\boldsymbol{\Pi}}$ at $\boldsymbol{\omega}$. When a particular local parametrisation $\sigma$ for $\Omega_{0}$ is chosen with $\mathbf{p} \in \mathbb{R}^{4 I+11}$ satisfying $\sigma(\mathbf{p})=\boldsymbol{\omega}, \mathrm{d} \overline{\boldsymbol{\Pi}}_{\boldsymbol{\omega}}$ can be identified with the Jacobian matrix of the composite mapping $\overline{\boldsymbol{\Pi}} \circ \sigma$ at $\mathbf{p}$. For a given linear map $\mathbf{A}$, let $\mathcal{R}(\mathbf{A})$ and $\mathcal{N}(\mathbf{A})$ denote the range and null spaces of $\mathbf{A}$, respectively. The dimension of $\bar{\Pi}\left(\Omega_{0}\right)$ is the same as the dimension of $\mathcal{R}\left(\mathrm{d} \overline{\boldsymbol{\Pi}}_{\boldsymbol{\omega}}\right)$ at a generic $\omega$; this is basically because the dimension of a manifold is the same as the dimension of the tangent space to the manifold at any particular point. On the other hand,

$$
\operatorname{dim} \mathcal{N}\left(\mathrm{d} \overline{\boldsymbol{\Pi}}_{\boldsymbol{\omega}}\right)+\operatorname{dim} \mathcal{R}\left(\mathrm{d} \overline{\boldsymbol{\Pi}}_{\boldsymbol{\omega}}\right)=\operatorname{dim} T_{\boldsymbol{\omega}}\left(\Omega_{0}\right),
$$

where $T_{\boldsymbol{\omega}}\left(\Omega_{0}\right)$ denotes the tangent space of $\Omega_{0}$ at $\boldsymbol{\omega}$. At the level of the Jacobian matrix, this is just an instance of the rank-nullity result of linear algebra saying that the nullity (the dimension of the null of space) and the rank of a matrix add up to the number of columns of the matrix. The dimension of $T_{\boldsymbol{\omega}}\left(\Omega_{0}\right)$ equals the dimension of $\Omega_{0}$ and this, in view of the constraint (4), equals $4 I+11$, one less than the dimension of $\Omega$. Thus to establish that $\operatorname{dim} \bar{\Pi}\left(\Omega_{0}\right) \geq 4 I+7$ we need only show that $\operatorname{dim} \mathcal{N}\left(\mathrm{d} \overline{\boldsymbol{\Pi}}_{\boldsymbol{\omega}}\right) \leq 4$ at a generic $\boldsymbol{\omega}$.

Let

$$
\delta \boldsymbol{\omega}=\left(\delta \mathbf{A}, \delta \mathbf{b}, \delta \mathbf{v}_{1}, \ldots, \delta \mathbf{v}_{I}, \delta w_{1}, \ldots, \delta w_{I}\right)
$$

be a tangent vector to $\Omega_{0}$ at $\boldsymbol{\omega}$. In view of (4), we have

$$
\mathbf{b}^{\top} \delta \mathbf{b}=0 .
$$

For $\delta \boldsymbol{\omega}$ to fall into the null space of $\mathrm{d} \overline{\boldsymbol{\Pi}}_{\boldsymbol{\omega}}$, it is necessary and sufficient that

$$
\mathrm{d}\left(\boldsymbol{\Pi}_{i}\right)_{\boldsymbol{\omega}}(\delta \boldsymbol{\omega})=\delta w_{i} \mathbf{A}+w_{i} \delta \mathbf{A}+\delta \mathbf{b v}_{i}^{\top}+\mathbf{b} \delta \mathbf{v}_{i}^{\top}=\mathbf{0}
$$

for each $i=1, \ldots, I$. Assume that $\delta \boldsymbol{\omega}$ is in $\mathcal{N}\left(\mathrm{d} \overline{\boldsymbol{\Pi}}_{\boldsymbol{\omega}}\right)$ so that (6) holds. Pre-multiplying (6) by $\mathbf{b}^{\top}$ and using (4) and (5) yields

$$
\delta w_{i} \mathbf{b}^{\top} \mathbf{A}+w_{i} \mathbf{b}^{\top} \delta \mathbf{A}+\delta \mathbf{v}_{i}^{\top}=\mathbf{0} .
$$

Pre-multiplying in turn this equation by $\mathbf{b}$ and subtracting the resulting equation from (6) leads to

$$
\delta w_{i}\left(\mathbf{I}_{3}-\mathbf{b b}^{\top}\right) \mathbf{A}+w_{i}\left(\mathbf{I}_{3}-\mathbf{b b}^{\top}\right) \delta \mathbf{A}+\delta \mathbf{b v}_{i}^{\top}=\mathbf{0} .
$$

The latter formula can be rewritten as

$$
\left(\mathbf{I}_{3}-\mathbf{b} \mathbf{b}^{\top}\right)\left(\delta w_{i} \mathbf{A}+w_{i} \delta \mathbf{A}\right)+\delta \mathbf{b v}_{i}^{\top}=\mathbf{0},
$$

which upon post-multiplying by $\mathbf{v}_{i}$ gives

$$
\left(\mathbf{I}_{3}-\mathbf{b} \mathbf{b}^{\top}\right)\left(\delta w_{i} \mathbf{A}+w_{i} \delta \mathbf{A}\right) \mathbf{v}_{i}+\delta \mathbf{b}\left\|\mathbf{v}_{i}\right\|^{2}=\mathbf{0} .
$$

Hence

$$
\delta \mathbf{b}=-\left(\mathbf{I}_{3}-\mathbf{b b}^{\top}\right)\left(\delta w_{i} \mathbf{A}+w_{i} \delta \mathbf{A}\right)\left\|\mathbf{v}_{i}\right\|^{-2} \mathbf{v}_{i} .
$$

Plugging this expression for $\delta \mathbf{b}$ back into (8), we find that

$$
\left(\mathbf{I}_{3}-\mathbf{b b}^{\boldsymbol{\top}}\right)\left(\delta w_{i} \mathbf{A}+w_{i} \delta \mathbf{A}\right)\left(\mathbf{I}_{3}-\left\|\mathbf{v}_{i}\right\|^{-2} \mathbf{v}_{i} \mathbf{v}_{i}^{\top}\right)=\mathbf{0} .
$$

This can equivalently be restated as

$$
\left(\mathbf{I}_{3}-\mathbf{b b}^{\top}\right)\left(\frac{\delta w_{i}}{w_{i}} \mathbf{A}-\delta \mathbf{A}\right) \mathbf{P}_{\mathbf{v}_{i}}^{\perp}=\mathbf{0},
$$

where

$$
\mathbf{P}_{\mathbf{v}_{i}}^{\perp}=\mathbf{I}_{3}-\left\|\mathbf{v}_{i}\right\|^{-2} \mathbf{v}_{i} \mathbf{v}_{i}^{\top} .
$$

Generically, given a pair $i$ and $j$ of distinct indices, the vectors $\mathbf{v}_{i}$ and $\mathbf{v}_{j}$ are linearly independent and their cross product $\mathbf{v}_{i} \times \mathbf{v}_{j}$ is non-zero. Since

$$
\mathbf{v}_{i}^{\top}\left(\mathbf{v}_{i} \times \mathbf{v}_{j}\right)=\mathbf{v}_{j}^{\top}\left(\mathbf{v}_{i} \times \mathbf{v}_{j}\right)=\mathbf{0},
$$


we have

$$
\mathbf{P}_{\mathbf{v}_{i}}^{\perp}\left(\mathbf{v}_{i} \times \mathbf{v}_{j}\right)=\mathbf{P}_{\mathbf{v}_{j}}^{\perp}\left(\mathbf{v}_{i} \times \mathbf{v}_{j}\right)=\mathbf{v}_{i} \times \mathbf{v}_{j} .
$$

In view of (10),

$$
\left(\mathbf{I}_{3}-\mathbf{b b}^{\top}\right)\left(\frac{\delta w_{i}}{w_{i}} \mathbf{A}-\delta \mathbf{A}\right)\left(\mathbf{v}_{i} \times \mathbf{v}_{j}\right)=\mathbf{0}
$$

and

$$
\left(\mathbf{I}_{3}-\mathbf{b b}^{\top}\right)\left(\frac{\delta w_{j}}{w_{j}} \mathbf{A}-\delta \mathbf{A}\right)\left(\mathbf{v}_{i} \times \mathbf{v}_{j}\right)=\mathbf{0} .
$$

Subtracting the second of these equations from the first, we obtain

$$
\left(\frac{\delta w_{i}}{w_{i}}-\frac{\delta w_{j}}{w_{j}}\right)\left(\mathbf{I}_{3}-\mathbf{b b}^{\top}\right) \mathbf{A}\left(\mathbf{v}_{i} \times \mathbf{v}_{j}\right)=\mathbf{0}
$$

Generically, the vector $\left(\mathbf{I}_{3}-\mathbf{b b}^{\top}\right) \mathbf{A}\left(\mathbf{v}_{i} \times \mathbf{v}_{j}\right)$ is non-zero, so

$$
\frac{\delta w_{i}}{w_{i}}=\frac{\delta w_{j}}{w_{j}} .
$$

In other words, the $\delta w_{i} / w_{i}$ 's have a common value. Denote this value by $\delta \lambda$. Then (10) can be rewritten as

$$
\left(\mathbf{I}_{3}-\mathbf{b b}^{\top}\right)(\delta \lambda \mathbf{A}-\delta \mathbf{A}) \mathbf{P}_{\mathbf{v}_{i}}^{\perp}=\mathbf{0} .
$$

We now show that in fact

$$
\left(\mathbf{I}_{3}-\mathbf{b b}^{\top}\right)(\delta \lambda \mathbf{A}-\delta \mathbf{A})=\mathbf{0}
$$

It suffices to prove that

$$
\left(\mathbf{I}_{3}-\mathbf{b b}^{\top}\right)(\delta \lambda \mathbf{A}-\delta \mathbf{A}) \mathbf{x}=\mathbf{0}
$$

for each length-3 vector $\mathbf{x}$. Choose two linearly independent vectors from amongst the $\mathbf{v}_{i}$ 's, say, $\mathbf{v}_{1}$ and $\mathbf{v}_{2}$. As any length-3 vector is a linear combination of $\mathbf{v}_{1}, \mathbf{v}_{2}$, and $\mathbf{v}_{1} \times \mathbf{v}_{2}$, (13) will be established once it is shown that it holds for $\mathbf{x}$ equal to $\mathbf{v}_{1}, \mathbf{v}_{2}$, and $\mathbf{v}_{1} \times \mathbf{v}_{2}$. Since $\mathbf{P}_{\mathbf{v}_{1}}^{\perp}\left(\mathbf{v}_{1} \times \mathbf{v}_{2}\right)=\mathbf{v}_{1} \times \mathbf{v}_{2}$, it follows from (11) that

$$
\begin{aligned}
& \left(\mathbf{I}_{3}-\mathbf{b b}^{\top}\right)(\delta \lambda \mathbf{A}-\delta \mathbf{A})\left(\mathbf{v}_{1} \times \mathbf{v}_{2}\right) \\
& \quad=\left(\mathbf{I}_{3}-\mathbf{b b}^{\top}\right)(\delta \lambda \mathbf{A}-\delta \mathbf{A}) \mathbf{P}_{\mathbf{v}_{1}}^{\perp}\left(\mathbf{v}_{1} \times \mathbf{v}_{2}\right)=\mathbf{0}
\end{aligned}
$$

so (13) holds in the case $\mathbf{x}=\mathbf{v}_{1} \times \mathbf{v}_{2}$. Now

$$
\mathbf{v}_{1}=\left(1-\frac{\left(\mathbf{v}_{1}^{\top} \mathbf{v}_{2}\right)^{2}}{\left\|\mathbf{v}_{1}\right\|^{2}\left\|\mathbf{v}_{2}\right\|^{2}}\right)^{-1}\left(\frac{\mathbf{v}_{2}^{\top} \mathbf{v}_{1}}{\left\|\mathbf{v}_{2}\right\|^{2}} \mathbf{P}_{\mathbf{v}_{1}}^{\perp} \mathbf{v}_{2}+\mathbf{P}_{\mathbf{v}_{2}}^{\perp} \mathbf{v}_{1}\right)
$$

as direct verification shows. Using this representation together with (11) yields immediately

$$
\left(\mathbf{I}_{3}-\mathbf{b b}^{\top}\right)(\delta \lambda \mathbf{A}-\delta \mathbf{A}) \mathbf{v}_{1}=\mathbf{0}
$$

By interchanging the roles of $\mathbf{v}_{1}$ and $\mathbf{v}_{2}$ in the above argument,

$$
\left(\mathbf{I}_{3}-\mathbf{b b}^{\top}\right)(\delta \lambda \mathbf{A}-\delta \mathbf{A}) \mathbf{v}_{2}=\mathbf{0} .
$$

Thus (13) also holds also in the cases $\mathbf{x}=\mathbf{v}_{1}$ and $\mathbf{x}=\mathbf{v}_{2}$.

As an immediate consequence of (12),

$$
\begin{aligned}
\delta \mathbf{A} & =\mathbf{b b}^{\top} \delta \mathbf{A}+\left(\mathbf{I}_{3}-\mathbf{b b}^{\top}\right) \delta \mathbf{A} \\
& =\mathbf{b b}^{\top} \delta \mathbf{A}+\delta \lambda\left(\mathbf{I}_{3}-\mathbf{b b}^{\top}\right) \mathbf{A} .
\end{aligned}
$$

Let $\delta \mathbf{c}$ be the length- 3 vector defined by $\delta \mathbf{c}=\delta \mathbf{A b}$. Then

$$
\delta \mathbf{A}=\mathbf{b}(\delta \mathbf{c})^{\top}+\delta \lambda\left(\mathbf{I}_{3}-\mathbf{b b}^{\top}\right) \mathbf{A},
$$

expressing $\delta \mathbf{A}$ linearly in terms of $\delta \mathbf{c}$ and $\delta \lambda$. The relation

$$
\delta w_{i}=w_{i} \delta \lambda
$$

expresses $\delta w_{i}$ linearly in terms of $\delta \lambda$. Now (9) in which $\delta \mathbf{A}$ and $\delta w_{i}$ are replaced by the right-hand sides of (14) and (15), respectively, gives an expression for $\delta \mathbf{b}$ that is linear in $\delta \mathbf{c}$ and $\delta \lambda$. Finally, (7) rewritten as

$$
\delta \mathbf{v}_{i}=-\delta w_{i} \mathbf{A}^{\top} \mathbf{b}-w_{i}(\delta \mathbf{A})^{\top} \mathbf{b}
$$

and combined with (14) and (15) as in the previous step gives an expression for $\delta \mathbf{v}_{i}$ that is linear in $\delta \mathbf{c}$ and $\delta \lambda$. Thus all components of $\delta \boldsymbol{\omega}$ depend linearly on $\delta \mathbf{c}$ and $\delta \lambda$, which shows that the null space of $\mathrm{d} \overline{\boldsymbol{\Pi}}_{\boldsymbol{\omega}}$ is at most four dimensional.

We complete this section with a brief recapitulation of the logic behind our main dimensionality result. The fact that $\operatorname{dim} \mathcal{N}\left(\mathrm{d} \overline{\boldsymbol{\Pi}}_{\boldsymbol{\omega}}\right) \leq 4$ implies that $\operatorname{dim} \mathcal{R}\left(\mathrm{d} \overline{\boldsymbol{\Pi}}_{\boldsymbol{\omega}}\right) \geq 4 I+7$, and hence that $\operatorname{dim} \overline{\boldsymbol{\Pi}}\left(\Omega_{0}\right) \geq 4 I+7$. This in conjunction with the final result of Section 4 implies that $\operatorname{dim} \mathcal{H}=$ $4 I+7$.

\section{Cardinality of the explicit constraints}

The fact that $\operatorname{dim} \mathcal{H}=4 I+7$ has an immediate implication as to the number of underlying explicit constraints. This number is exactly equal to $5 I-7$. It is calculated as the difference between the dimension of the space of all $9 \times I$ matrices and $\operatorname{dim} \mathcal{H}$.

We note in passing that the bulk of the explicit constraints determining $\mathcal{H}$ as a subset of $\mathbb{R}^{9 \times I}$ can easily be identified in the case where $I \geq 5$. In fact, using (3), we see that any $\mathbf{H}$ satisfies

$$
\mathbf{H}=\mathbf{S T} \text {, }
$$

where $\mathbf{S}$ is the $9 \times 4$ matrix given by

$$
\mathbf{S}=\left[\mathbf{I}_{3} \otimes \mathbf{b}, \mathbf{a}\right]
$$

and $\mathbf{T}$ is the $4 \times I$ matrix given by

$$
\mathbf{T}=\left[\begin{array}{lll}
\mathbf{v}_{1} & \cdots & \mathbf{v}_{I} \\
w_{1} & \cdots & w_{I}
\end{array}\right]
$$




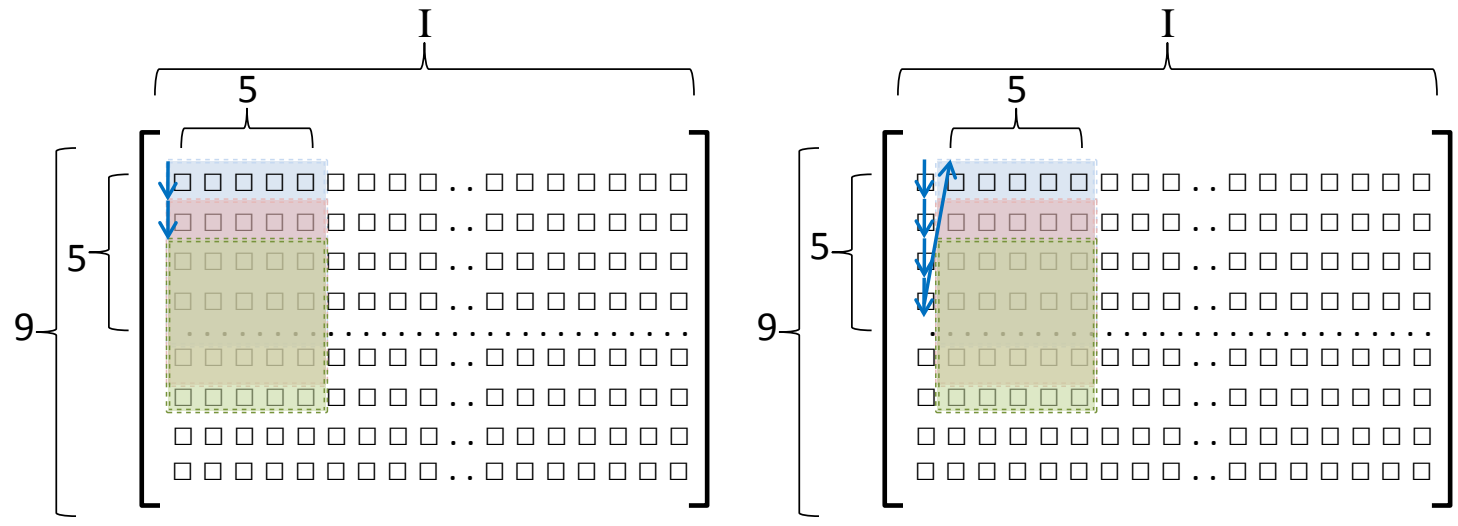

Figure 2. Scheme of selection of minors.

It follows from (16) that, whenever $I \geq 4, \mathbf{H}$ has rank at most 4. In other words, $\mathcal{H} \subset \mathbb{R}_{4}^{9 \times I}$ for $I \geq 4$, this being the rank-four constraint mentioned in the introduction. Remembering that $\mathbb{R}_{k}^{m \times n}$ is a $k(m+n-k)$-dimensional variety in $\mathbb{R}^{m \times n}$, we realise that

$$
\operatorname{dim} \mathbb{R}_{4}^{9 \times I}=4(9+I-4)=4 I+20 .
$$

Thus-under the assumption that $I \geq 5$-to determine $\mathbb{R}_{4}^{9 \times I}$ as a subset of $\mathbb{R}^{9 \times I}$ in a generic way, a set of $9 I-(4 I+20)=5 I-20$ constraints is required.

One such set can be obtained by taking specific minor determinants of $\mathbf{H}$ of order 5 for defining functions of the constraints. We first pick the left upper minor of $\mathbf{H}$ of order 5 . We then select four more minors of $\mathbf{H}$ of order 5 by sliding down by one row at each new choice until the last ninth row of $\mathbf{H}$ is reached, all this happening within the range of the first four columns of $\mathbf{H}$. At this stage five minors of $\mathbf{H}$ of rank 5 are extracted. We then repeat the whole process starting this time with the upper minor of order 5 in the range between the second and sixth columns of $\mathbf{H}$. By additionally sliding down four times, another set of five minors of $\mathbf{H}$ of order 5 is obtained. Continuing repeatedly to slide down and shift to the right, we reach at the $(I-4)$ th round the last column of $\mathbf{H}$, and after the final slide-down the whole process halts. In this way, a total of $5(I-4)$ minors of $\mathbf{H}$ of order 5 is selected (see Figure 2).

With $5 I-20$ constraints determining $\mathbb{R}_{4}^{9 \times I}$ exhibited, we now need another $5 I-7-(5 I-20)=13$ constraints to finally determine $\mathcal{H}$. Intriguingly, this number of remaining constraints does not depend on the value of $I$.

\section{Conclusion and future work}

In this paper we have revealed the dimension of the set of all collections of interdependent homography matrices in the case when the homographies described by these matrices are induced by a fixed number of multiple planes in the $3 \mathrm{D}$ scene between two views. The number of the underlying explicit constraints has also been exhibited. Future work includes generalising these results to the case of collections of homography matrices induced by multiple planes between more than two views. Most interesting, however, is finding an avenue through which to specify the explicit constraints completely and succinctly.

\section{Acknowledgement}

This research was supported by the Australian Research Council.

\section{References}

[1] J. Bochnak, M. Coste, and M.-F. Roy. Real Algebraic Geometry. Springer, Berlin, 1998. 2

[2] P. Chen and D. Suter. Rank constraints for homographies over two views: revisiting the rank four constraint. Int. J. Computer Vision, 81(2):205-225, 2009. 1, 3

[3] W. Chojnacki, Z. L. Szpak, M. J. Brooks, and A. van den Hengel. Multiple homography estimation with full consistency constraints. In Proc. Digital Image Computing: Techniques and Applications Conf., pages 480-485, 2010. 1

[4] A. Eriksson and A. van den Hengel. Optimization on the manifold of multiple homographies. In Proc. IEEE 12th Int. Conf. Computer Vision Workshops, pages 242-249, 2009. 2, 3

[5] J. Harris. Algebraic Geometry. Springer, New York, 1995. 3

[6] R. I. Hartley and A. Zisserman. Multiple View Geometry in Computer Vision. Cambridge University Press, Cambridge, 2nd edition, 2004. 1

[7] O. Kähler and J. Denzler. Rigid motion constraints for tracking planar objects. In Proc. 29th DAGM Symposium, volume 4713 of Lecture Notes in Computer Science, pages 102-111, 2007. 1

[8] A. Shashua and S. Avidan. The rank 4 constraint in multiple ( $\geq 3$ ) view geometry. In Proc. 4th European Conf. Computer Vision, volume 1065 of Lecture Notes in Computer Science, pages 196-206, 1996. 1

[9] L. Zelnik-Manor and M. Irani. Multiview constraints on homographies. IEEE Trans. Pattern Anal. Mach. Intell., 24(2):214-223, 2002. 1 